\title{
Search for Leptoquarks and Lepton Flavour Violation with the H1 Experiment at HERA
}

\author{
Ana Dubak ${ }^{1,2}$ \\ 1- University of Montenegro, Faculty of Science \\ Cetinjski put bb, Podgorica, Montenegro \\ 2- Max-Planck-Institute for Physics \\ Föhringer Ring 6, Munich, Germany
}

\begin{abstract}
Recent results of searches for leptoquarks with the H1 experiment at HERA are presented. A search for first generation leptoquarks is performed using $e^{-} p$ data with longitudinally polarised electrons collected in the second phase of HERA running. A search for lepton flavour violating processes $e p \rightarrow \mu X$ and $e p \rightarrow \tau X$ is performed using $e^{+} p$ and $e^{-} p$ data from the first phase of HERA running collected from 1998 to 2000. No evidence for direct or indirect production of leptoquarks has been found. The results are interpreted in terms of limits on the Yukawa coupling of leptoquarks and lepton flavour violating processes.
\end{abstract}

\section{Introduction}

Leptoquarks (LQs) are colour triplet bosons which appear in various unifying theories beyond the Standard Model (SM). At HERA, leptoquarks could be singly produced by the fusion of the initial state electron ${ }^{\mathrm{a}}$ of energy $27.6 \mathrm{GeV}$ with a quark from the incoming proton of energy $920 \mathrm{GeV}$. The phenomenology of LQs is discussed in detail in [2]. This note presents a search for LQs coupling to first generation fermions in scattering of longitudinally polarised electrons on protons at a centre-of-mass energy of $\sqrt{s} \approx 320 \mathrm{GeV}$. For the lefthanded running phase with an average polarisation of $-27 \%$, the integrated luminosity amounts to $60 \mathrm{pb}^{-1}$, whereas for righthanded running phase with an average polarisation of $34 \%, 32 \mathrm{pb}-1$ of data are analysed.

The LQ concept can also be used to search for lepton flavour violation (LFV) processes in electron-proton collisions. In ep collisions at HERA, LFV processes $e p \rightarrow \mu X$ and $e p \rightarrow \tau X$ lead to final states with a muon or a tau and a hadronic system X. The search for LFV phenomena is performed in $e p$ collision data with unpolarised electrons recorded during the years 1998-2000 by the H1 experiment, corresponding to an integrated luminosity of $66.5 \mathrm{pb}^{-1}$ for $e^{+} p$ collisions and $13.7 \mathrm{pb}^{-1}$ for $e^{-} p$ collisions at a centre-of-mass energy $\sqrt{s}=319 \mathrm{GeV}$.

\section{Search for Leptoquarks}

Due to the more favourable quark-densities of quarks with respect to anti-quarks at high $x$, the $e^{-} p$ data sets are mostly sensitive to LQs with fermion number $F=|L+3 B|=2$. The search reported here considers the decays $\mathrm{LQ} \rightarrow e q$ and $\mathrm{LQ} \rightarrow \nu q$ where $q$ represents both quarks and anti-quarks. These LQ decays lead to final states similar to those of deepinelastic scattering (DIS) neutral current (NC) and charged current (CC) interactions at

\footnotetext{
an this note "electron" refers generically to both electrons and positrons. Where distinction is required the symbols $e^{+}$and $e^{-}$are used.
} 
very high $Q^{2}$, the negative four-momentum transfer squared. If the final state is of type $e q$, the LQ mass is reconstructed from the measured kinematics of the scattered electron. If the final state is of type $\nu q$, the LQ mass is reconstructed from the hadronic final state [2].

This search is based on inclusive $\mathrm{NC}$ and CC DIS data in the kinematic domain $Q^{2}>500 \mathrm{GeV}^{2}$ and $0.1<y<0.9$, where the inelasticity variable $y$ is defined as $y=Q^{2} / M^{2}$. The cuts on $y$ remove regions of poor reconstruction, poor resolution, large QED radiative effects and background from photoproduction processes. The selection of NC-like events requires an identified electron with transverse momentum above $11 \mathrm{GeV}$. The selection of CC-like events follows closely that presented in $[2,3]$. The missing transverse momentum is required
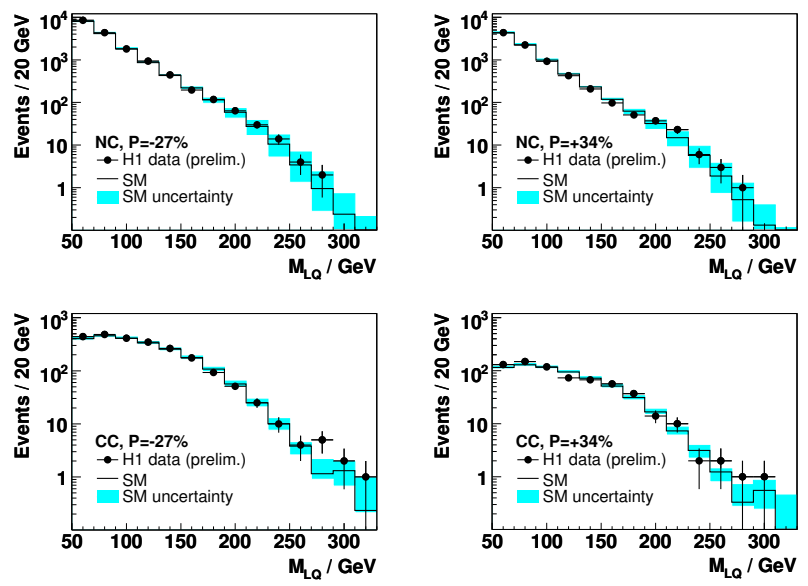

Figure 1: Mass spectra for the neutral current (up) and the charged current events (down).

The mass spectra measured for NC-like and CC-like events in the left- and righthanded data sets, shown in figure 1, are compared with the SM predictions, obtained using a MonteCarlo calculation [4] and the CTEQ5D parametrisation [5] for the parton densities. In all cases the data are well described by the SM prediction.

Since no evidence for LQ production is observed in either the $\mathrm{NC}$ or CC data samples, the data are used to set constraints on LQs which couple to first generation fermions.

The resulting constraints for the four scalar

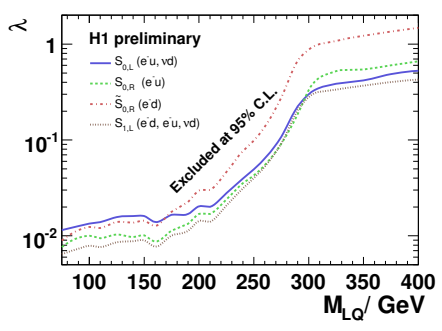

Figure 2: Exclusion limits for the 7 LQs with $F=2$. The limits are expressed at 95\% CL on the coupling $\lambda$ as a function of the leptoquark mass. Domains above the curves are excluded. and the three vector LQs with $F=2$ are shown in figure 2. The areas above the curves are excluded at 95\% CL. The strongest constraints on the coupling $\lambda$ can be set for LQ masses below the kinematic limit of the s-channel. At higher masses the production is no longer resonant, but rather contact interaction like and the cross section scales approximately with $\left(\lambda / M_{L Q}\right)^{4}$. For a coupling of electromagnetic strength $\left(\lambda=\sqrt{4 \pi \alpha_{e m}}=0.3\right)$ this analysis rules out LQ masses below 276 to $304 \mathrm{GeV}$, depending on the LQ type. 


\section{Search for Lepton Flavour Violation}

This analysis presents a search for LFV mediated by LQs with $F=0$ and $F=2$. For convenience only one LFV transition is considered: either between the first and the second generations $e p \rightarrow L Q \rightarrow \mu X$ or between the first and the third generations $e p \rightarrow L Q \rightarrow \tau X$.

To determine the signal detection efficiencies, events with LQs are generated using the LEGO [6] event generator with the CTEQ5L parametrisation of the parton distribution functions (PDF) of the proton [7]. The LQ signal expectation is a function of the LQ type, mass, coupling constant and branching ratio $\beta_{L F V}$. The contributions from SM background processes which may mimic the signal include NC and CC DIS, photoproduction, lepton pair production and real $\mathrm{W}$ boson production.

LQs with couplings to the first and second generation leptons can be produced in ep collisions and may decay to a muon and a quark. The signature is an isolated high $P_{T}$ muon backto-back to the hadronic system in the transverse plane. In general, a muon deposits a very small fraction of its energy in the LAr calorimeter. The signal is therefore expected to exhibit large $P_{T}^{c a l o}$, which is the net transverse momentum reconstructed from all clusters recorded in the LAr calorimeter alone. A detailed description of selection criteria is presented in [8].

Leptoquarks with couplings to the first and the third lepton generation can be produced in $e p$ collisions and may decay to a tau and a quark. Tau leptons are identified using the electronic, muonic and hadronic decays of the tau. The final state resulting from the electronic tau decay, $\tau \rightarrow e \nu_{e} \nu_{\tau}$, leads to an event topology that is very similar to that of high $Q^{2}$ NC DIS events. The preselection follows that presented in [2]. Muonic tau decays $\tau \rightarrow \mu \nu_{\mu} \nu_{\tau}$ result in similar final states as the high $P_{T}$ muon signatures of muonic LQ decay. Therefore the same selection cuts are applied here. The hadronic decays of the high $P_{T}$ tau lead to a typical signature of a high high $P_{T}$ "pencil-like" jet. The signal topology is a di-jet event with no leptons. The final selection criteria for all three tau decay channels are given in [8].
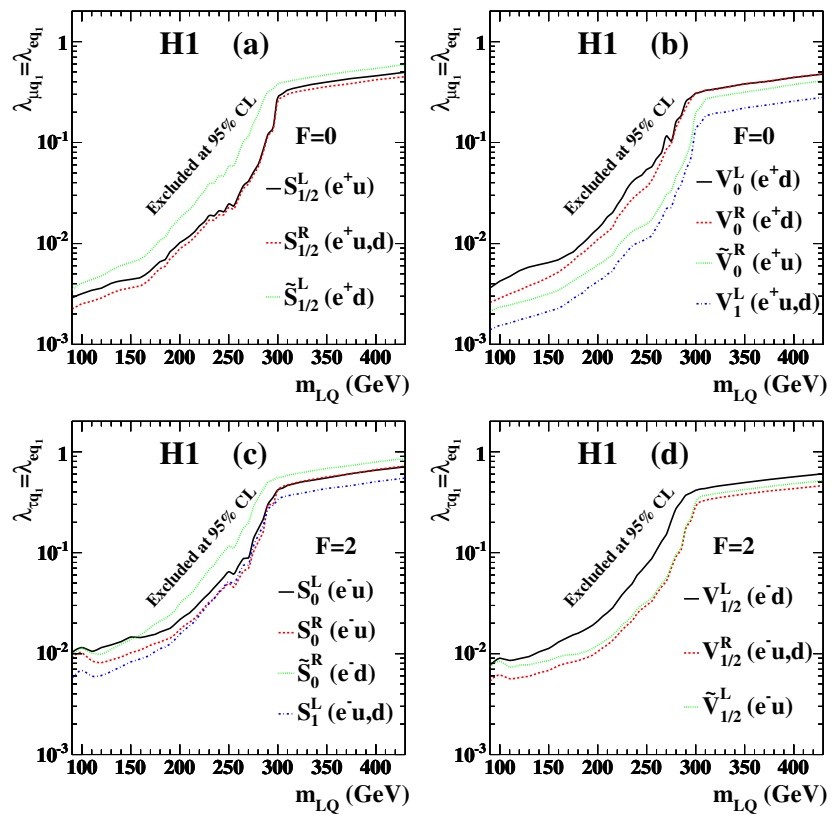

Figure 3: Comparison of limits at 95\% CL on the coupling constants $\lambda_{l q}$ assuming $\lambda_{\mu q}=\lambda_{e q}(\mathrm{a}, \mathrm{b})$ and $\lambda_{\tau q}=\lambda_{e q}(\mathrm{c}, \mathrm{d})$ as a function of the LQ mass $m_{L Q}$. The areas above the lines represent the excluded regions.

No candidate is found in the fi-

nal data sample of the muon channel nor in the electronic and muonic tau decay channel. One event is selected in hadronic tau decay channel which is in agreement with the SM expectation. 
The results of the search are interpreted in terms of exclusion limits on the mass and the coupling of LQs that may mediate LFV. In order to cover the full LQ decay width and to generalise the results of LFV searches in $e p$ collisions to an arbitrary weight between the lepton flavour conservation (LFC) and LFV decay channels, the searches for LFC decays presented in [9] are combined with each of the LFV search channels $\mu X$ or $\tau X$ of the present analysis.

Upper limits on the coupling $\lambda_{\mu q}$ and $\lambda_{\tau q}$ of all 14 LQ types to a muon-quark pair and a tau-quark pair, respectively, are determined as a function of the LQ mass. Assuming lepton universality the couplings $\lambda_{\mu q}$ and $\lambda_{\tau q}$ are taken to be equal to $\lambda_{e q}$. Examples of such a limits are shown in figure 3 .

Figure 4 shows an example of excluded regions for one LQ type in the $\lambda_{l q_{1}}-\lambda_{e q_{1}}$ plane for the case of an arbitrary decay rate between the LFC and LFV decay channels, $\beta_{L F V}$. For $\beta \gg 0.05\left(\lambda_{l q} \gg \lambda_{e q}\right)$ the present analysis extends significantly the published limits on $\lambda_{e q}$ to lower values.

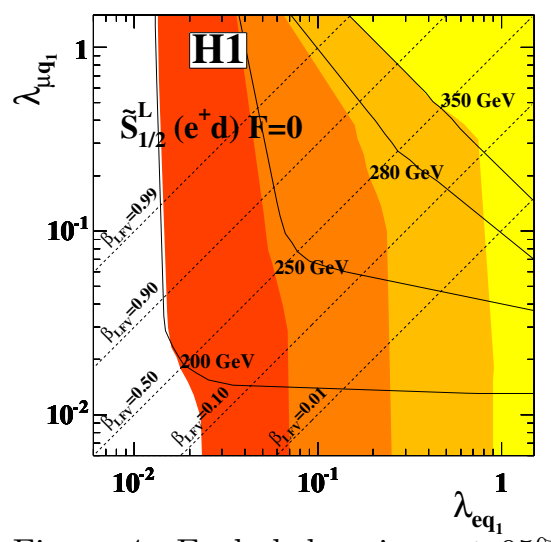

Figure 4: Excluded regions at $95 \%$ CL (filled) in $\lambda_{l q_{1}}-\lambda_{e q_{1}}$ plane for four different leptoquark masses. The bounds deduced without the combination with first generation LQs are shown as black curves corresponding to the different LQ masses.

\section{Conclusion}

A search for LQs with fermion number $\mathrm{F}=2$ has been performed using the polarised $e^{-} p$ data recorded by $\mathrm{H} 1$ in 2005. No signal has been observed and constraints on leptoquarks have been set, which for F $=2$ LQs extend beyond the domains excluded previously by H1. For a coupling of electromagnetic strength, LQ masses below $276-304 \mathrm{GeV}$ can be ruled out, depending on the LQ type.

No signal for the LFV processes $e p \rightarrow \mu X$ or $e p \rightarrow \tau X$ is found. Constraints on LFV LQ couplings are set combining the LFV search with the search for first generation LQs. Assuming a coupling of electromagnetic strength, leptoquarks mediating lepton flavour violating processes $e \rightarrow \mu$ and $e \rightarrow \tau$ can be ruled out up to masses of $459 \mathrm{GeV}$ and $379 \mathrm{GeV}$, respectively.

\section{References}

[1] Slides: http://indico. cern.ch/contributionDisplay $\cdot$ py? contribId=129\&sessionId=9\&conf Id=9499

[2] C. Adloff et al., Eur. Phys. J. C11 447 (1999) [Erratum-ibid. C14 553 (1999)], [hep-ex/9907002].

[3] C. Adloff et al., Eur. Phys. J. C19 269 (2001), [hep-ex/0012052].

[4] DJANGO 6.2; G.A. Schuler and H. Spiesberger, Proc. of the Workshop Physics at HERA, W. Buchmüller and G. Ingelman (Editors), (October 1991, DESY-Hamburg) Vol. 3 p. 1419.

[5] H. L. Lai et al., Eur. Phys. J. C12 375 (2000), [hep-ph/9903282].

[6] K. Rosenbauer, dissertation RWTH Aachen (in German), PITHA 95/16, July 1995.

[7] J. Pumplin et al., JHEP 0207012 (2002), [hep-ph/0201195]

[8] A. Aktas et al., submitted to Eur. Phys. J. C, [hep-ex/0703004]

[9] A. Aktas et al., Phys. Lett. B629 9 (2005), [hep-ex/0506044]. 